Marquette University

e-Publications@Marquette

$1-1-2002$

Rapid, Specific Determination of Iodine and Iodide by Combined Solid-Phase Extraction/Diffuse Reflectance Spectroscopy

Matteo Arena

Marquette University, matteo.arena@marquette.edu

Marc D. Porter

Iowa State University - Ames

James S. Fritz

Iowa State University - Ames

Accepted version. Analytical Chemistry, Vol. 74, No. 1 (2002): 185-190. DOI. (C) 2002 American Chemical Society. Used with permission.

Matteo P. Arena was affiliated with Iowa State University, Ames at the time of publication. 
Marquette University

e-Publications@Marquette

Finance Faculty Research and Publications/College of Business

Administration

This paper is NOT THE PUBLISHED VERSION; but the author's final, peer-reviewed manuscript. The published version may be accessed by following the link in the citation below.

Analytical Chemistry, Vol. 74, No. 1 (2002): 185-190. DOI. This article is @ American Chemical Society and permission has been granted for this version to appear in e-Publications@Marquette. American Chemical Society does not grant permission for this article to be further copied/distributed or hosted elsewhere without the express permission from American Chemical Society.

\section{Rapid, Specific Determination of lodine and lodide by Combined Solid-Phase Extraction/Diffuse Reflectance Spectroscopy}

Matteo P. Arena

Microanalytical Instrumentation Center, Ames Laboratory - USDOE Department of Chemistry, lowa State University

Marc D. Porter

Microanalytical Instrumentation Center, Ames Laboratory - USDOE

Department of Chemistry, lowa State University

James S. Fritz

Microanalytical Instrumentation Center, Ames Laboratory - USDOE

Department of Chemistry, lowa State University 


\section{Abstract}

A new, rapid methodology for trace analysis using solid-phase extraction is described. The two-step methodology is based on the concentration of an analyte onto a membrane disk and on the determination by diffuse reflectance spectroscopy of the amount of analyte extracted on the disk surface. This method, which is adaptable to a wide range of analytes, has been used for monitoring ppm levels of iodine and iodide in spacecraft water. lodine is used as a biocide in spacecraft water. For these determinations, a water sample is passed through a membrane disk by means of a 10-mL syringe that is attached to a disk holder assembly. The disk, which is a polystyrene-divinylbenzene composite, is impregnated with poly(vinylpyrrolidone) (PVP), which exhaustively concentrates iodine as a yellow iodine-PVP complex. The amount of concentrated iodine is then determined in only $2 \mathrm{~s}$ by using a hand-held diffuse reflectance spectrometer by comparing the result with a calibration curve based on the Kubelka-Munk function. The same general procedure can be used to determine iodide levels after its facile and exhaustive oxidation to iodine by peroxymonosulfate (i.e., Oxone reagent). For samples containing both analytes, a two-step procedure can be used in which the iodide concentration is calculated from the difference in iodine levels before and after treatment of the sample with peroxymonosulfate. With this methodology, iodine and iodide levels in the 0.1-5.0 ppm range can be determined with a total workup time of $\sim 60 \mathrm{~s}$ with a RSD of $\sim 6 \%$.

Because of its speed and effectiveness, solid-phase extraction (SPE) has become the preferred technique for concentrating analytes prior to their analysis by chromatography or by other techniques. Its increasing popularity is underscored not only by a review that listed more than 350 papers on SPE in $1999^{1}$ but also by the publication of several books. ${ }^{2-5}$ Much of the earlier work in SPE employed particle-packed cartridges. More recently, the technique has expanded to include the use of particleloaded membrane disks, which offers the advantage of greater extraction efficiency and smaller size. Solid-phase microextraction (SPME) with a polymer-coated fiber as the adsorbent is also becoming increasingly popular for preconcentration of organic analytes prior to gas chromatographic analysis. In most cases, the concentrated analytes are eluted from the extractant particles for further analyses, usually by means of an organic solvent, or, in SPME, by thermal desorption.

It is also possible to eliminate postextraction analysis by quantifying the amount of an extracted analyte directly on the extraction disk. Thus, the amount of a colored analyte can be measured on the surface of the disk by diffuse reflectance spectroscopy. This "readout" method is rapid and convenient and eliminates the need for an elution step and any postextraction workup of eluted analytes. Moreover, the applicability of this strategy can be readily expanded by impregnating the membrane disk with a reagent that can chemically react with an analyte to produce a colored complex.

One way to perform a determination of this type involves a dynamic equilibrium between an analyte in the sample solution and a membrane disk. The most basic version of this approach is to immerse a strip of reagent-treated paper into a liquid sample and estimate the concentration of the analyte from the intensity of the immobilized colorimetric complex. However, the amount of analyte concentrated by the extractive solid is dependent on immersion time, with equilibrium usually reached only after long immersion times.

Higher concentration factors can be obtained when the sample solution is mixed with the colorimetric reagent and then passed through the membrane disk. This scheme has been used by several laboratories to preconcentrate colored species prior to their quantification by diffuse reflectance 
spectroscopy. Preformed complexes of silver and palladium ions ${ }^{6}$ and zinc ions ${ }^{7}$ have been preconcentrated on a disk or cloth impregnated with vinylpyridine groups. An assortment of reagentimpregnated papers has been used for colorimetric determination of several heavy metals after pulling the sample through the test papers. ${ }^{8}$ Active chlorine in water has been determined colorimetrically after a color-forming reaction with toluidine groups on polyurethane films. ${ }^{9}$ Moreover, phosphate has been determined by a molybdenum blue method, ${ }^{10}$ uranium(VI) as a thiocyanate complex, ${ }^{11}$ and cobalt and platinum ions as pyridylazonaphthol (PAN) complexes. ${ }^{12}$ In the case of the latter, the limit of detection was 100 times lower than for determinations that quantified the concentration of the PAN complexes directly in solution.

This paper presents a simple, reliable methodology that we have named colorimetric solid-phase extraction (C-SPE). In C-SPE, a liquid sample is pulled through a small extraction disk that contains embedded polymeric particles and is impregnated with a suitable reagent. The color formed by the resulting complex is then measured by a hand-held diffuse reflectance spectrophotometer directly on the disk surface. This methodology has been developed for monitoring 0.1-5.0 ppm levels of iodine, which is used as a biocide in the storage and distribution systems for drinking water on spacecraft. In contrast to the previously cited work, the extraction disk in our approach is preimpregnated with the complexing reagent. Importantly, preimpregnation eliminates the need for mixing the complexing reagent with the sample solution prior to extraction onto the membrane. Moreover, the use of a commercially available, hand-held diffuse reflectance spectrometer enables the quantitative determination of the extracted analyte in $\sim 2 \mathrm{~s}$. These two features provide significant ease and rapidity to the method, which we believe is the first to link the exhaustive concentration of an analyte by SPE to its quantitation by diffuse reflectance spectroscopy.

\section{Experimental Section}

Reagents and Chemicals. All solutions were prepared with deionized water that was further purified by a NANOpure water purification system (Barnstead).

Standard lodine Solution. The standard iodine stock solution (200.0 ppm) was prepared by diluting $1.515 \mathrm{~mL}$ of iodine volumetric $0.1041 \mathrm{~N}$ standard solution (Aldrich) in $100.0 \mathrm{~mL}$ (class A volumetric flask) of water. Standard iodine solutions with lower concentrations were then prepared by the appropriate dilution of this stock.

Standard lodide Solution. The standard iodide stock solution (1000 ppm) was prepared by dissolving $0.131 \mathrm{~g}$ of potassium iodide (Aldrich) in $100.0 \mathrm{~mL}$ (class A volumetric flask) of water. More dilute standard iodine solutions were prepared from this stock.

Poly(vinylpyrrolidone) (PVP) Solution. The PVP solution used to impregnate the membranes was prepared by dissolving $3 \mathrm{~g}$ of PVP powder (average $M_{\mathrm{w}} \sim 10000$, Aldrich) in $0.100 \mathrm{~L}$ (class A volumetric flask) of a 1:1 water/methanol solution.

Oxone Solution. The Oxone solution (i.e., prepackaged potassium peroxymonosulfate) was prepared by dissolving $0.1 \mathrm{~g}$ of Oxone (Aldrich) in $0.100 \mathrm{~L}$ of water.

SPE Membranes. The extraction membranes were prepared by impregnating 3M Empore SDB-XC (polystyrene-divinylbenzene) 47-mm extraction disks and 3M Empore C18 47-mm extraction disks with PVP. This process involved mounting a membrane on a Millipore 47-mm all-glass vacuum filter holder (Millipore) and pulling $10 \mathrm{~mL}$ of the PVP solution through the membrane by vacuum. Residual solution 
was then removed by vacuum for an additional 1-2 min. After these steps were completed, the membranes were cut into 13-mm disks by means of a cork borer.

Instrumentation. A BIK-Gardner color-guide sphere $\mathrm{d} / 8^{\circ}$ diffuse reflectance spectrophotometer (model LCB-6830, BIK-Gardner) was used to collect all of the spectral data from the membrane materials. This hand-held spectrophotometer is small, light in weight, and battery operated and can collect reflectance data over the entire visible spectral range $(400-700 \mathrm{~nm})$ in 20-nm increments in only $1.5 \mathrm{~s}$. The entire spectrum in displayed on the instrument readout panel. The small aperture of the instrument enables reflectance readings to be made on a 13-mm-diamater disk. The samples are mated to the spectrophotometer by means of the sample positioning housing of the instrument.

The 13-mm membranes were mounted in 13-mm plastic Swinnex filter holders (Catalog No. SX00 0013 00 , Millipore). A $10-\mathrm{mL}$ plastic syringe containing $10.0 \mathrm{~mL}$ of sample was connected to the holder in order to extract the analyte from the sample by its passage through the membrane.

Software. The spectrophotometer was interfaced to a PC by using a serial cable. The spectra were transferred to the PC and downloaded to the color-guide MS-Excel worksheet for the instrument in order to plot the reflectance data from 400 to $700 \mathrm{~nm}$. The data were then transferred to another Excel worksheet to calculate the Kubelka-Munk functions and plot calibration curves.

Extraction Procedure and Readout of Extracted lodine and lodide. The 10-mL plastic syringe was loaded with $10 \mathrm{~mL}$ of water sample. The 13-mm membrane, previously impregnated with PVP, was placed on the plastic support screen of the syringe holder and the holder was tightly screwed. The 10$\mathrm{mL}$ glass syringe was loaded with $10 \mathrm{~mL}$ of water sample. The syringe was connected to the holder via a Luer Lock fitting and the sample was injected manually throughout the membrane; the extraction step required $\sim 0.5 \mathrm{~min}$. After the extraction, the holder was removed from the syringe and unscrewed. The membrane was removed from the holder and placed under the sample area locator, the spectrophotometer was placed on the membrane, and the trigger was pushed to acquire the spectrum of the iodine retained on the membrane surface.

\section{Results and Discussion}

Principles of Colorimetric SPE. In C-SPE, an aqueous sample is pulled through an extraction disk impregnated with a reagent that simultaneously concentrates the desired analyte and enables the development of a color due to the complexation of the analyte with the reagent. The amount of colored analyte complex that is affixed on the outer portion of the disk is then determined by a handheld diffuse reflectance spectrophotometer. The present application of C-SPE is designed to provide a rapid, reliable way to monitor $0.1-5 \mathrm{ppm}$ levels of iodine or iodide (after its oxidation to iodine) in the drinking water of spacecraft. All of the iodine is retained by a solid-phase extraction mechanism as a yellow complex on a polystyrene-divinylbenzene membrane that was previously impregnated with PVP. lodide can be determined by the same procedure after its oxidation to iodine. The method is extremely facile and rapid; i.e., the $10-\mathrm{mL}$ sample is pushed through the disk in $\sim 30 \mathrm{~s}$ and the reflectance measurements take only $\sim 2 \mathrm{~s}$. The total workup time for a sample, which includes mating the membrane to the spectrometer, is then $\sim 60 \mathrm{~s}$.

C-SPE is basically a variant of a conventional solid-phase extraction procedure. To our knowledge, this investigation marks the first time that iodine has been concentrated from aqueous samples by SPE. In previous SPE methods, the extracted analytes were eluted from the extractant particles for further analysis. The elution step is usually accomplished by means of an organic solvent or, in SPME, by 
thermal desorption. The present method is quicker and more convenient than traditional SPE in that the extracted analyte is measured directly on the membrane surface by reflectance spectrophotometry, eliminating the need for an elution step.

The performance criteria for the effective implementation of C-SPE are somewhat more severe than those necessary for the more conventional modes of SPE. Both methods require a high retention factor so that essentially all of the analyte will be retained on the SPE disk. However, C-SPE, when used with diffuse reflectance spectroscopy, requires that the analyte be confined on or near the upper surface of the disk. The requirement arises from the fact that diffuse reflectance spectroscopy has a limited depth sensitivity. ${ }^{13}$ Therefore, any portion of the analyte retained on the disk below the light penetration depth will not be detected, which will negatively affect sensitivity. Being a colorimetric technique, CSPE also requires that the analyte or the resulting complex be a chromophore.

Diffuse Reflectance Measurements. The Kubelka-Munk (K-M) equation provides an effective approach for quantitavely relating the observed signal to the sample concentration for diffuse reflectance measurements. ${ }^{14}$ The $\mathrm{K}-\mathrm{M}$ function, $F(R)$, is defined as

$$
F(R)=(1-R)^{2} / 2 R
$$

where $R$ is the absolute diffuse reflectance. This function is directly related to the concentration of an absorbing sample species, $C$, by

$$
F(R)=\epsilon C / s
$$

where $\varepsilon$ is the molar absorbivity of the sample species and $s$ is the scattering coefficient of the sample surface. The $\mathrm{K}-\mathrm{M}$ equation was used in order to quantify the amount iodine extracted on the surface of the membrane, noting that $F(R)$ is proportional to the surface concentration of the adsorbed iodine either as free iodine or as the iodine-PVP complex.

lodine Preconcentration. Because of its hydrophobicity, molecular iodine is quantitatively extracted from water upon passing an aqueous sample through the polymeric membrane without the presence of an impregnated complexating reagent. lodine is strongly retained on the topmost portion of the membrane. The diffuse reflectance spectrum of extracted iodine is shown in Figure 1, curve A. Quantitation of the extracted iodine is complicated by its volatility; iodine gradually evaporates if much time elapses between the extraction and readout steps unless the disk is sealed in a small volume.

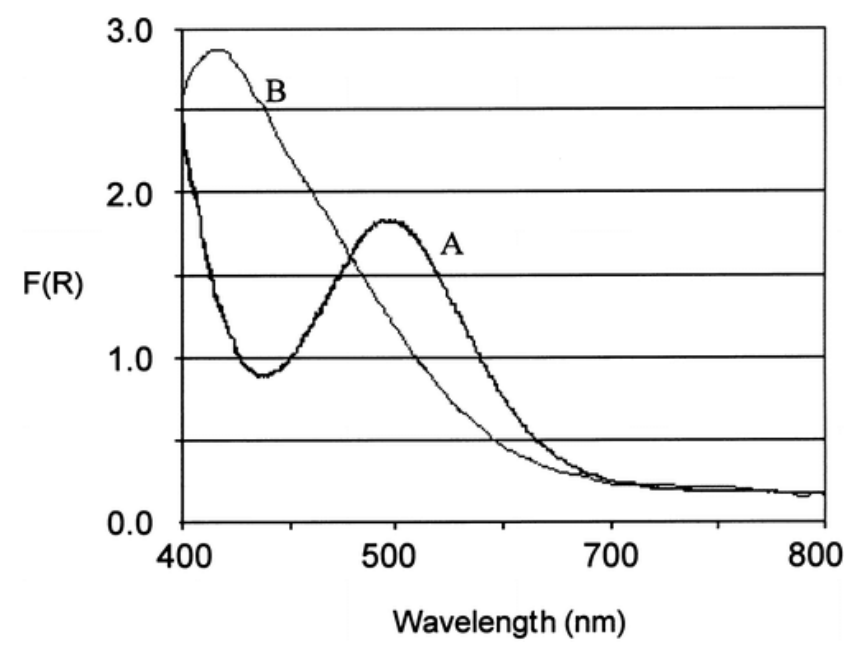

Figure 1 lodine reflectance spectra. 
To reduce the difficulty associated with volatility, the procedure was modified by treating the disk with PVP. The iodine is retained by the membrane disk as the iodine-PVP complex. ${ }^{15}$ The resulting complex has the two benefits of being less volatile and more intensely colored than molecular iodine. The $F(R)$ spectrum of the iodine-PVP complex (Figure 1, curve B) has a maximum at $420 \mathrm{~nm}$ that is significantly higher than the maximum of the iodine at $500 \mathrm{~nm}$. PVP-impregnated disks provide higher sensitivity, and therefore, they were used in all subsequent experiments.

To identify the optimal amount of PVP that can be incorporated into the polymeric membrane for the effective extraction and determination of iodine, six SDB-XC membranes were treated with $10 \mathrm{~mL}$ of solutions of varied PVP levels $(5,10,20,30,40$, and $50 \mathrm{~g} / \mathrm{L})$. The membranes were then used in extractions of a $3.0 \mathrm{ppm}$ iodine solution and characterized by using diffuse reflectance spectroscopy. A $\mathrm{K}-\mathrm{M}$ plot of the responses at $440 \mathrm{~nm}$ revealed that the value of $F(R)$ increased from 0.6 for the membrane treated with the $5 \mathrm{~g} / \mathrm{L}$ PVP solution to almost 0.75 for the $20 \mathrm{~g} / \mathrm{L}$ PVP solution and reached a constant value of $\sim 0.8$ for the 30,40 , and $50 \mathrm{~g} / \mathrm{L}$ PVP solutions. This dependence is attributed to the saturation of the membrane by the impregnating solution having higher PVP levels. For this reason, all the membranes used for the subsequent experiments were treated with $10 \mathrm{~mL}$ of a $30 \mathrm{~g} / \mathrm{L} \mathrm{PVP}$ solution.

Plotting and Quantitation. To develop a suitable calibration curve, reflectance measurements were made at several wavelengths after passing 10-mL samples of standard 0.1-5.0 ppm iodine in water through the impregnated disks. A plot of $F(R)$ versus iodine concentration gave a higher slope at 420 $\mathrm{nm}$ between 0.1 and $1 \mathrm{ppm}$ but leveled off at higher concentrations. The best linearity for a calibration plot from 0.1 to $5.0 \mathrm{ppm}$ was obtained at $440 \mathrm{~nm}$. The resulting calibration plot is shown in Figure 2 and, after a regression analysis, has a correlation factor of 0.9996 between 0.0 and 5.0 ppm.

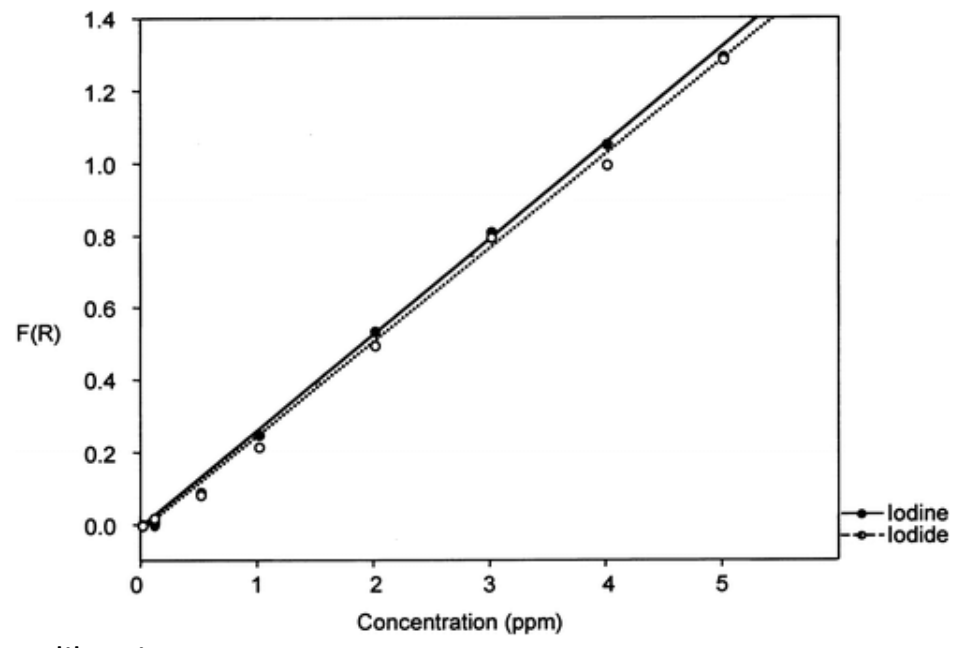

Figure 2 lodine and iodide calibration curves.

For each concentration, five sample solutions were pulled through five separate membranes. The different points in the graph correspond to the average of the five replicates. The interval of confidence varies from a maximum of $\pm 9.7 \%$ for $0.1 \mathrm{ppm}$ to a minimum of $\pm 6.0 \%$ for $5 \mathrm{ppm}(n=5, p=$ 0.95). The detection limit, calculated by adding 3 times the standard deviation of the blank to the average of the blank, is $0.05 \mathrm{ppm}$.

Determination of lodide. In aqueous solution, iodine and iodide coexist through the following equilibrium:

$$
\mathrm{I}_{2}+\mathrm{I}^{-} \rightleftarrows \mathrm{I}_{3}^{-}
$$


Tests showed, however, that, for the iodine concentration range of importance (1.0-5.0 ppm), the presence of iodide, even in considerable excess, did not detectably affect the iodine determination by our method. This result is consistent with the low value for the formation constant of the cited equilibrium $\left(7.00 \times 10^{2}\right) \cdot{ }^{16}$

A standard method for analysis of iodide in water and wastewater involves its oxidation to iodine with Oxone reagent. ${ }^{17}$ The same process was applied to determine iodide by colorimetric-SPE by adding 100 $\mu \mathrm{L}$ of a $1 \mathrm{~g} / \mathrm{L}$ Oxone solution in $10 \mathrm{~mL}$ of water sample, which instantaneously oxidized iodide to iodine in the $\mathrm{pH}$ range between 4.0 and 7.5 .

By using Oxone, it is feasible to analyze mixtures of iodine and iodide in samples containing low concentrations of each. lodine is determined first; after oxidation by Oxone, the sum of iodine plus iodide is measured. The amount of iodide in the sample is then calculated from the difference in the two values.

The oxidation of iodide by Oxone is almost complete between $\mathrm{pH} 4.0$ and 5.0, but the reflectance drops sharply between $\mathrm{pH} 6.5$ and 7.0. All the subsequent iodide determinations were therefore carried out at $\mathrm{pH}$ 5. Figure 2 shows a linear calibration plot for iodide that is slightly below and nearly parallel to that of iodine; the difference between the two calibrations is not significant. Data for the analysis of aqueous samples containing ppm concentrations of both iodine and iodide are summarized in Table 1. The results are within the $95 \%$ interval of confidence.

Table 1. Analysis of lodine and lodide Mixtures Using Oxone To Oxidize lodide to lodine

\begin{tabular}{|l|l|l|l|l|}
\hline \multicolumn{2}{|l|}{ sample composition, $\mathrm{ppm}$} & \multicolumn{4}{|l|}{ composition found, $\mathrm{ppm}$} \\
\hline $\mathrm{I}_{2}$ & $\mathrm{I}^{-}$ & $\mathrm{I}_{2}$ & $\mathrm{I}^{-}$ & $\mathrm{I}_{2}+\mathrm{I}^{-}$ \\
\hline 4.5 & & 4.5 & & \\
\hline & 4.5 & & & 4.3 \\
\hline 0.5 & 4.0 & 0.5 & 3.9 & 4.4 \\
\hline 4.0 & 0.5 & 3.9 & 0.6 & 4.5 \\
\hline
\end{tabular}

Interferences. Experiments were also carried out to determine the effect of foreign ions on the quantitation of iodide in aqueous samples after oxidation with Oxone. The interference of chloride, bromide, and fluoride was studied because they also belong to the halogen group and might also be extracted by the polymeric membrane generating interference. Nitrate and nitrite were also studied because they are present in significant concentrations in drinking water. Results for $1 \mathrm{ppm}$ iodide solutions with different ions after oxidation with Oxone are presented in Table 2. Ppm concentrations of chloride, nitrate, nitrite, and fluoride do not interfere in the analysis (the interval of confidence for five repetitions of a $1 \mathrm{ppm}$ iodine solution is $\pm 7.5 \%$; therefore, a difference of $8.0 \%$ is inside the prediction limits and is considered acceptable). Only bromide interferes at high concentration (10 ppm) because the bromide is oxidized to bromine, which is colored and is extracted by the membrane disk. The Kubelka-Munk value for bromine is $\sim 50$ times lower than that of iodine, which explains why only high concentrations of bromine interfere. 
Table 2. Effect of Foreign lons on the C-SPE Determination of 1 ppm lodide

\begin{tabular}{|l|l|l|l|l|}
\hline added ion & reflectance (\%) & $F(R)$ & error (\%) & interference \\
\hline none & 53.5 & 0.202 & & \\
\hline chloride $5 \mathrm{ppm}$ & 53.0 & 0.208 & +3 & no \\
\hline bromide $1 \mathrm{ppm}$ & 52.2 & 0.218 & +8 & no \\
\hline bromide $10 \mathrm{ppm}$ & 48.9 & 0.266 & +32 & yes \\
\hline fluoride $2 \mathrm{ppm}$ & 52.3 & 0.217 & +8 & no \\
\hline nitrite $0.5 \mathrm{ppm}$ & 53.8 & 0.198 & -2 & no \\
\hline nitrate $2 \mathrm{ppm}$ & 52.3 & 0.218 & +8 & no \\
\hline
\end{tabular}

Sodium chloride does not interfere even when present in high concentrations. A 1.0 ppm solution of iodide gave the same reflectance reading when $2 \mathrm{~g} / \mathrm{L}$ and even $33 \mathrm{~g} / \mathrm{L}(\mathrm{NaCl}$ concentration in seawater) sodium chloride was added to the water sample.

Comparison with Alternative Methods. A distinction needs to be made between C-SPE and the traditional analytical technique in which a reagent-impregnated material is simply immersed into the sample solution to test for a given analyte (this approach is also used for the SPME extraction step). When a PVP-impregnated disk was placed into $20 \mathrm{~mL}$ of a $5 \mathrm{ppm}$ iodine solution while the solution was vigorously stirred, more than 60 min was required to reach equilibrium; moreover, the final $F(R)$ value was 0.77 compared to $F(R)=1.97$ by the C-SPE method. This experiment demonstrated that the C-SPE procedure provides a superior sensitivity complemented by a greater rapidity.

The method by which PVP is added to the sample solution prior to the extraction step was also investigated briefly. In the concentration of metal-organic complexes onto a solid-phase extractant, the most effective extractions have usually been obtained when the reagent is premixed with the sample solution prior to the extraction step. In the present system, the opposite was found to be true. The sample solution was prepared by mixing $1 \mathrm{ppm}$ iodine and $30 \mathrm{~g} / \mathrm{L}$ PVP in $10 \mathrm{~mL}$ of a 50:50 water/methanol solution. The use of methanol is required to dissolve the high quantity of PVP required by the method. However, methanol also elutes the PVP-iodine complex from the membrane. For this reason, the signal produced by this $1 \mathrm{ppm}$ iodine solution is much lower $(F(R)=0.05)$ than that produced when $1 \mathrm{ppm}$ iodine is extracted by a membrane pretreated with PVP $(F(R)=0.22)$. This result further illustrates the inherent merits of the new concept.

Effect of Experimental Variables. Membrane Material. Two other types of membrane disks were considered for use in applying C-SPE to the determination of iodine: an Empore membrane loaded with sulfonated polystyrene-divinylbenzene particles, and an Empore membrane loaded with C-18 silica particles. The former has a pale yellow color that interferes with the detection of the color of the PVP-iodine complex and was therefore not considered to be a viable candidate for further experiments. The latter, however, is white, retains iodine after impregnation with PVP, and gives a linear calibration plot. However, the slope of the calibration curve for the C18-silica disk showed a sensitivity that was $30 \%$ lower that of the SDB-XC disk. This difference is ascribed to a higher extraction efficiency of the SDB-XC membrane, which is a consequence of the high affinity between the polystyrene-divinylbenzene particles of the membrane and PVP. Thus, the loading of PVP is greater in the SDB-XC membrane than in the C-18 silica, which is composed of alkyl chains that have a lower affinity for aromatic materials such as PVP. 
Iodine Evaporation. Reflectance measurements at $440 \mathrm{~nm}$ on PS-DVB disks pretreated with PVP were made as a function of time after the extraction step. Figure 3 shows a gradual linear decline in reflectance with respect to elapsed time before measurement. The slope is steeper at higher concentrations of iodine. This effect can be attributed to a slow sublimation of iodine from the membrane surface even when it is complexed by PVP. This behavior suggests that the iodine is extracted as a thin layer on the uppermost surface of the disk. The iodine sublimation does not affect the analysis because, as mentioned before, the measurement by the hand-held spectrometer takes $2 \mathrm{~s}$.

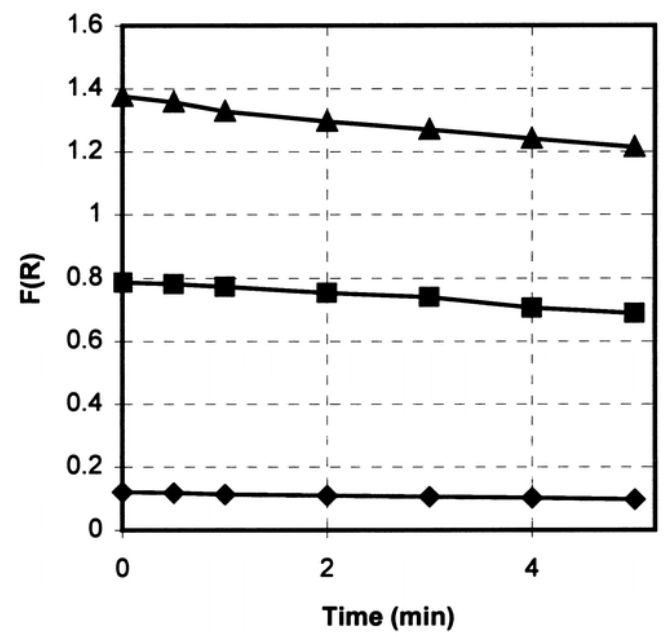

Figure $3 F(R)$ versus time elapsed from sample extraction to measurement $(0.5(\diamond), 3(-)$, and 5 (可) ppm iodine solution).

Shelf Life of PVP-Impregnated Disks. Experiments in which a set of disks impregnated with PVP were prepared and tested once a week for 6 months indicated that long-term storage has no detrimental effect on the results. All the determinations of a $3 \mathrm{ppm}$ iodine solution carried out in different weeks were within the $95 \%$ interval of confidence.

Temperature. The temperature of the water sample has a significant effect on the observed value of $F(R)$. After an initial increase in $F(R)$ between 4 and $10^{\circ} \mathrm{C}$, there is a steady decrease between 10 and $33^{\circ} \mathrm{C}$. For a $0.5 \mathrm{ppm}$ iodine solution, $F(R)$ is 0.16 at $4{ }^{\circ} \mathrm{C}$ and increases to 0.24 at $10{ }^{\circ} \mathrm{C}$ but steadily decreases at higher temperatures: $F(R)=0.20$ at $15^{\circ} \mathrm{C}, F(R)=0.17$ at $23{ }^{\circ} \mathrm{C}$, and $F(R)=0.13$ at $33^{\circ} \mathrm{C}$. This downward trend is due to the increasing rate of sublimation of iodine from the membrane as the temperature increases. To eliminate the effect of this variable, all the experiments were carried out at the constant temperature of $23^{\circ} \mathrm{C}$.

Organic Solvent in Sample Solution. The effect of the presence of an organic solvent in the aqueous samples was examined by mixing methanol with water samples in various proportions. An almost linear increase of $20 \%$ in $F(R)$ for samples containing $2.0 \mathrm{ppm}$ iodine was observed passing from 0 to $20 \%$ methanol. Thus, the interfacial transfer efficiency of iodine appears to be favored by the presence of a modest amount of methanol. However, the $F(R)$ decreased sharply from 0.9 at $20 \%$ methanol to 0.1 at $50 \%$ methanol. The extraction equilibrium is shifted toward the liquid phase by stronger solvation of iodine by the methanol/water solution that also partially washed the PVP from the membrane.

Disk Capacity. The retention capacity of a PVP-treated disk for iodine was determined by passing an aqueous sample containing 100 ppm iodine through the disk until breakthrough was detected. A 32-mg disk (13-mm diameter) was able to retain $\sim 1 \mathrm{mg}(3.0 \%)$ of iodine. 
$p H$. Initially, distilled water spiked with iodine was used to simulate drinking water on spacecraft. When ordinary drinking water samples containing various salts were investigated, the need to add $\mathrm{pH}$ buffers became evident. The dependence of $F(R)$ on $\mathrm{pH}$ is plotted in Figure 4.

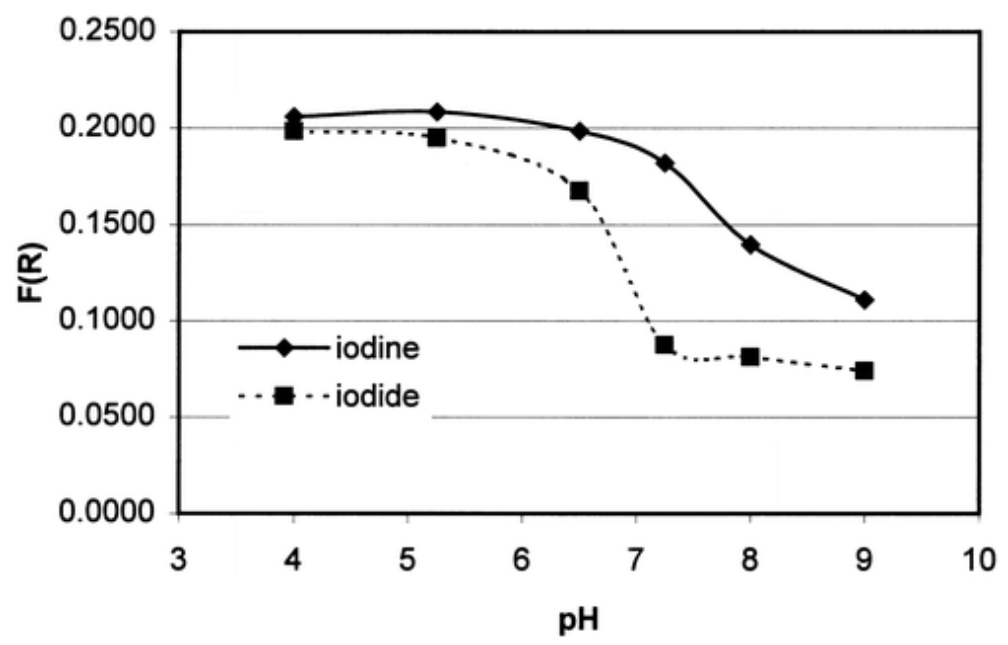

Figure 4 Effect of $\mathrm{pH}$ on the determination of a $1 \mathrm{ppm}$ iodine sample and a $1 \mathrm{ppm}$ iodide sample.

The signal is almost constant between $\mathrm{pH} 4.0$ and 6.5, but it decreases at higher $\mathrm{pH}$ values. Samples buffered at $\mathrm{pH} 5.0$ were used in the subsequent experiments.

\section{Conclusions}

Colorimetric-SPE is an integrated analytical method that allows determining low concentrations of iodine, iodide, or both. A high concentration factor is obtained by extraction of the selected analytes onto an impregnated membrane disk containing embedded polymer particles. No solvent elution or thermal desorption is required because the amount of the colored analyte complex is directly measured on the disk by reflectance spectrophotometry.

The flow-through mode of SPE used here provides a much greater concentration effect and hence better sensitivity than methods in which a color species is measured in solution or on the surface of a probe that is merely dipped into the sample solution.

The method is also very rapid. The concentration step requires $\sim 30 \mathrm{~s}$ and the reflectance measurement only $2 \mathrm{~s}$. The equipment is small, portable, and battery operated so that analyses can be performed anywhere.

The potential of this system is enormous because this method is by no means limited to the determination of iodine and iodide. It should be applicable to the determination of any analyte that is colored or forms a colored complex and can be extracted onto the surface of a membrane disk.

\section{Acknowledgment}

Insightful discussions with Paul Mudgett, Jeff Rutz, and John Shultz are acknowledged. This work was supported by NASA under contract NAG91191, and by the Microanalytical Instrumentation Center of lowa State University. The Ames Laboratory is operated for the U.S. Department of Energy by lowa State University under contract W-7405-eng-82. 


\section{References}

1. Fritz., J. S.; Macka, M. J. Chromatogr., A 2000, 902, 137-166.

2. Thurman, E. M.; Mills, M. S. Solid-Phase Extraction-Principle and Practice; Wiley-Interscience: New York, 1998.

3. Pawliszyn, J. Solid-Phase Microextraction; Wiley: New York, 1997.

4. Fritz, J. S. Analytical Solid-Phase Extraction; Wiley-VCH: New York, 1999.

5. Simpson, N. J. K. Solid-Phase Extraction: Principles, Techniques, and Applications; Marcel Dekker: New York, 2000.

6. Matsuhisa, K.; Ozeki, K. Analyst 1986, 685.

7. Waki, H.; Korkisch, J. Talanta 1983, 30, 95-100.

8. Ivanov, V. M.; Kuznetsova, O. V.; Grineva, O. V. J. Anal. Chem. 1999, 54, 233-236.

9. Gur'eva, R. F.; Savvin, S. B. J. Anal. Chem. 2000, 55, 249-254.

10. Shvoeva, O. P.; Dedkova, V. P.; Savvin, S. B. J. Anal. Chem. 1998, 53, 1028-1032.

11. Amelin, V. G. J. Anal. Chem. 1999, 54, 574-581.

12. Dmitrienko, S. G.; Sviridova, O. A.; Pyatkova, L. N.; Zhukova, V. A.; Zolotov, Yu. A. Anal. Chim. Acta 2000, 405, 231-237.

13. Wendlandt, W. W.; Hecht, H. G. Reflectance Spectroscopy; Interscience Publishers: New York, 1996; pp 23-24.

14. Kortum, G. Reflectance Spectroscopy: Principles, Methods, Applications; Springer-Verlag: New York, 1969; pp 106-116.

15. Kader, A.; Ahmad, S.; El-Gendy, A. E.; Ek-Naga, N. H. Bull. Fac. Pharm. (Cairo Univ.) 1994, 32, 17-23.

16. Katzin, L. I.; Elizabeth, G. J. Am. Chem. Soc. 1955, 77, 5814-19.

17. Standard Methods for the Examination of Water and Wastewater, 19th ed.; WEF, AWWA, APHA: Washington, DC, 1995; p 4.73. 